\title{
Identification of Occupational Cancer Risks in British Columbia, Canada: A Population-Based Case-Control Study of 1,155 Cases of Colon Cancer
}

\section{Raymond Fang $^{1,2, *}$, Nhu Le ${ }^{2}$ and Pierre Band ${ }^{2,3}$}

1 American Academy of Physician Assistants, Suite 1300, 2318 Mill Road, Alexandria, VA 22314, USA

2 Cancer Control Research, British Columbia Cancer Agency, 675 West 10th Avenue, Vancouver, BC V5Z 1L3, Canada; E-Mails: nle@bccrc.ca (N.L.); pierre_band@hc-sc.gc.ca (P.B.)

3 Health Canada, 50 Columbine Drive, Tunney's Pasture, Ottawa, ON K1A 0K9, Canada

* Author to whom correspondence should be addressed; E-Mail: rfang@aapa.org; Tel.: +1-571-319-4327; Fax: +1-571-319-4328.

Received: 8 August 2011; in revised form: 2 September 2011 / Accepted: 19 September 2011 / Published: 26 September 2011

\begin{abstract}
Objective: Cancer has been recognized to have environmental origin, but occupational cancer risk studies have not been fully documented. The objective of this paper was to identify occupations and industries with elevated colon cancer risk based on lifetime occupational histories collected from 15,463 incident cancer cases. Method: A group matched case-control design was used. All cases were diagnosed with histologically proven colon cancers, with cancer controls being all other cancer sites, excluding rectum, lung and unknown primary, diagnosed at the same period of time from the British Columbia Cancer Registry. Data analyses were done on all 597 Canadian standard occupation titles and 1,104 standard industry titles using conditional logistic regression for matched data sets and the likelihood ratio test. Results: Excess colon cancer risks was observed in a number of occupations and industries, particularly those with low physical activity and those involving exposure to asbestos, wood dusts, engine exhaust and diesel engine emissions, and ammonia. Discussion: The results of our study are in line with those from the literature and further suggest that exposure to wood dusts and to ammonia may carry an increased occupational risk of colon cancer.
\end{abstract}


Keywords: occupational cancer; colon cancer; cancer risk; occupational exposure; carcinogen

\section{Introduction}

Colon cancer is one of the leading cancer causes of death. In Canada, colon cancer is the third most common form of cancer (after prostate cancer and lung cancer in men and breast cancer and lung cancer in women) and the third leading cancer cause of death (after lung cancer and prostate cancer in men and lung cancer and breast cancer in women) [1].

Despite the common occurrence of colon cancer, its etiology is not well established; age, family history [2], lifestyle factors [3-6] including daily alcohol use, low-fiber diets, a high consumption of animal fat and red meat, physical inactivity [7-10], obesity [11-13] and smoking [14] are reported risk factors. The evidence regarding occupational exposures related to the occurrence of colon cancer is generally limited and/or not consistent [15]. Exposures to asbestos [16,17], dyes in textile industry [18], hydrazine in rocket fuels [19], pesticides in agriculture [20], engine exhaust and diesel engine emissions [21] and occupations as managers [22], male farmers [23], painters, police, guards and firemen, motor transport workers and clerks as well as exposures in non-metallic mineral products, leather goods, meat, poultry, and fish products industries [24] have been reported to be associated with a higher risk of colon cancer. A consistent relationship, however, has primarily been found for physically inactive occupations [25-29].

We have, as part of a program aimed at detecting occupational risk factors in British Columbia, Canada, collected lifetime occupational histories from 15,463 incident cancer cases, of which 1,155 had a diagnosis of colon cancer. Occupational risks for this group of patients are presented in this article by assessing risk difference in comparison with internal controls consisting of all other cancer patients excluding lung and rectum cancer and cancer of unknown primary sites.

\section{Materials and Methods}

\subsection{General Methodology}

The general methodology of the study has been described in detail in a previous article [30]. Briefly, male cancer patients aged 20 years and older ascertained by the population-based British Columbia Cancer Registry (BCCR) for the years 1983 to 1990 inclusively, received a self-administered questionnaire requesting lifetime job descriptions, occupation and industry titles, and duration and period of work, as well as information on ethnic origin, education, and lifetime smoking habits. Questions about lifetime consumption of alcoholic beverages, which were initially omitted, were added to the questionnaire during the first year of the study. In the event of a patient's death, information was requested from the spouse or closest living relative. For the first two years, questionnaires were sent to all cases. Data collection continued until 1,000 completed questionnaires were accrued for each tumor site, or until 31 December 1990, whichever occurred first. The distribution of patients' usual occupations and usual industries as well as assessments of response bias 
and of questionnaire validation and reliability was conducted and previously reported [30]. The anatomic site and pathology of the primary tumor were coded using the 9th revision of the International Classification of Diseases [31] and the International Classification of Diseases for Oncology [32], respectively. Occupations and industries were coded according to the Canadian Standard Occupational Classification (SOC) and the Canadian Standard Industrial Classification (SIC), respectively $[33,34]$. In the SOC, occupations are coded according to two-digit major group codes, three-digit minor group codes, and four-digit unit group codes. In the SIC, industries are coded according to two-digit major group codes, three-digit group codes, and four-digit class codes. During the data-collection period, questionnaires were sent to all 25,726 eligible male cancer cases ascertained by the BCCR and 15,463 (60.1\%) were returned. Histological confirmation of diagnosis was obtained in all cases. For colon cancer, questionnaires were sent to 1,768 eligible cases, of which 1,156 (65.4\%) were returned.

\subsection{Statistical Methodology}

A group matched case-control design was used. Cases comprised all 1,156 colon cancer patients. Controls comprised internal controls with all other cancer sites, excluding lung cancer $(\mathrm{n}=2,998)$, rectum cancer $(n=1,095)$ and cancers of unknown primary site $(n=708)$, matched to the cases on exact age and year of diagnosis. These were based on a few methodological considerations. First, using other cancers as controls has a few advantages over population controls with regard to recall and interviewer bias when using general population controls, a mixture of live and deceased controls can be expected when selecting other cancer patients as controls and there are logistical and cost advantages in using other cancer patients as controls. Thus the study design using cancers as controls is likely to be used increasingly in the future, particularly in cancer registry settings [35]. Next, since lung cancer is mainly caused by smoking, excluding it from the control group would avoid potential bias of having too many smokers in controls. In addition, matching on age and year of diagnosis is a way to control for major confounders. Including year of diagnosis is a control of time when rapid changes in population structure were happened from 1960s through 1990s.

Data were analyzed using conditional logistic regression for matched sets and the likelihood ratio test [36,37] in a two-step procedure. In step 1, the effect of the following non-occupational confounding variables was assessed: marital status; education (less than 8 years; 8 to 11 years; high school; post-secondary); smoking (starting age at smoking, average number of cigarettes, pipe or cigars smoked per day, total years smoked); alcohol consumption (starting age at consuming alcohol; average of number of bottles of beer, glasses of wine, ounces of spirits consumed per day; total years of consuming alcohol); and person who filled out the questionnaire (self or proxy). Variables were selected in a forward fashion each being examined separately; Potentially important confounders $(P<0.2)$ were then included in the model and the remaining ones were examined; the process was repeated until no factor with $P<0.2$ was identified. In step 2, each occupation and industry was assessed separately using conditional logistic regression in which all significant confounding variables identified in step 1 were taken into account. The cut point of 0.20 was chosen in step 1 to ensure that the process has sufficient power to identify potentially important confounders. 
Matched case-control analyses were carried out using PECAN software [38], test of significance of the adjusted odds ratios (ORs), p-values and 95\% confidence intervals were calculated and presented. Analyses were performed for the following two different estimates of occupational exposures: ever occupation/industry (whether a job was ever held in a given occupation/industry under consideration) versus never, and usual occupation/industry (job with the longest held lifetime employment in a given occupation/industry). People in either ever or usual occupation/industry categories were always compared to their counterparts who were never in such occupation/industry. Due to all 597 occupation titles and 1,104 industry titles were analyzed and small numbers of cases in many occupation/industry categories, we intended to maximally present results of the analyses in this article with occupations and industries as long as their number of cases is five and over.

\section{Results}

Matching resulted in 1,155 colon cancer cases having at least one matching control and 7,552 controls, leaving an overall control to case ratio of 6.5:1. Site distribution of controls is listed in Table 1.

Table 1. Cancer site distribution of 7,552 controls.

\begin{tabular}{ccc}
\hline Site & Controls & Percent \\
\hline lip & 99 & $1.3 \%$ \\
oral cavity & 390 & $5.2 \%$ \\
esophagus & 174 & $2.3 \%$ \\
stomach & 343 & $4.5 \%$ \\
liver & 38 & $0.5 \%$ \\
pancreas & 136 & $1.8 \%$ \\
larynx & 280 & $3.7 \%$ \\
soft tissue sarcoma & 101 & $1.3 \%$ \\
melanoma & 458 & $6.1 \%$ \\
non-melanoma skin & 1,050 & $13.9 \%$ \\
prostate & 1,366 & $18.1 \%$ \\
testis & 82 & $1.1 \%$ \\
bladder & 972 & $12.9 \%$ \\
kidney & 314 & $4.2 \%$ \\
brain & 159 & $2.1 \%$ \\
Hodgkin's Disease & 52 & $0.7 \%$ \\
non-Hodgkin's lymphoma & 417 & $5.5 \%$ \\
multiple myeloma & 118 & $1.6 \%$ \\
leukemia & 184 & $2.4 \%$ \\
other sites & 326 & $4.3 \%$ \\
multiple sites & 493 & $6.5 \%$ \\
Total & 7,552 & $100.0 \%$ \\
\hline
\end{tabular}

The characteristics of cases and controls are shown in Table 2. 
Table 2. Characteristics of cases and controls.

\begin{tabular}{|c|c|c|}
\hline \multirow{2}{*}{$\begin{array}{l}\text { Colon Cancer } \\
\text { Characteristics }\end{array}$} & Cases $(n=1,155)$ & Controls $(n=7,552)$ \\
\hline & No. $(\%)$ & No. $(\%)$ \\
\hline \multicolumn{3}{|l|}{ Year of diagnosis } \\
\hline 1983 & 218(18.9) & $2,674(35.4)$ \\
\hline 1984 & $254(22.0)$ & $1,660(22.0)$ \\
\hline 1985 & $259(22.4)$ & $1,302(17.2)$ \\
\hline 1986 & $246(21.3)$ & $1,010(13.4)$ \\
\hline 1987 & $178(15.4)$ & $906(12.0)$ \\
\hline \multicolumn{3}{|l|}{ Marital Status } \\
\hline Single & $56(4.8)$ & $352(4.7)$ \\
\hline Married/common law & $958(82.9)$ & $6,269(83.0)$ \\
\hline Widowed & $74(6.4)$ & $463(6.1)$ \\
\hline Separated/divorced & $54(4.7)$ & $405(5.4)$ \\
\hline Not answered & $13(1.1)$ & $63(0.8)$ \\
\hline \multicolumn{3}{|l|}{ Education } \\
\hline$\leq 7$ years & $136(11.8)$ & $842(11.1)$ \\
\hline $8-11$ years & $511(44.2)$ & $3,336(44.2)$ \\
\hline High school & $138(11.9)$ & $830(11.0)$ \\
\hline Post-secondary & $329(28.5)$ & $2,144(28.4)$ \\
\hline Not answered & $41(3.5)$ & $400(5.3)$ \\
\hline \multicolumn{3}{|c|}{ Alcohol consumption status } \\
\hline Never drinker & 137(11.9) & $738(9.8)$ \\
\hline Ever drinker & $890(77.1)$ & $5,456(72.2)$ \\
\hline Not answered & $128(11.1)$ & $1,358(18.0)$ \\
\hline \multicolumn{3}{|l|}{ Smoking duration, years } \\
\hline 0 & $282(24.4)$ & $1,501(19.9)$ \\
\hline $1-29$ & $379(32.8)$ & $2,026(26.8)$ \\
\hline $30-44$ & $284(24.6)$ & $2,216(29.3)$ \\
\hline $45+$ & $196(17.0)$ & $1,682(22.3)$ \\
\hline Not answered & $14(1.2)$ & $127(1.7)$ \\
\hline \multicolumn{3}{|l|}{ Cigarette Pack-Years } \\
\hline 0 & $282(24.4)$ & $1,501(19.9)$ \\
\hline $1-24$ & $348(30.1)$ & $2,039(27.0)$ \\
\hline $25-49$ & $271(23.5)$ & $2,012(26.6)$ \\
\hline$\geq 50$ & $200(17.3)$ & $1,582(20.9)$ \\
\hline Unknown & $54(4.7)$ & $418(5.5)$ \\
\hline \multicolumn{3}{|l|}{ Pipe smoking status } \\
\hline Non-Pipe Smoker & $1,098(95.1)$ & $7,053(93.4)$ \\
\hline Pipe Smoker & $57(4.9)$ & $499(6.6)$ \\
\hline \multicolumn{3}{|c|}{ Respondent to questionnaire } \\
\hline Patient & $930(80.5)$ & $5,955(78.9)$ \\
\hline Proxy & $199(17.2)$ & $1,403(18.6)$ \\
\hline Unknown & $26(2.3)$ & $194(2.6)$ \\
\hline
\end{tabular}


Statistically significant ORs were noted for the following variables: smoking duration and education level as shown in Table 3; the latter was mainly due to the unknown category.

Table 3. Odds Ratios (OR) for potentially important confounding variables/effect modifiers.

\begin{tabular}{lccccc}
\hline \multicolumn{1}{c}{ Confounding Variable } & Cases & Controls & OR & P-value & $\begin{array}{c}\text { 95\% Confidence } \\
\text { Interval (95\% CI) }\end{array}$ \\
\hline Smoking duration, years & & & & & \\
0 & 282 & 1,504 & 1.00 & & \\
$1-29$ & 379 & 2,026 & 1.05 & 0.60 & $0.87-1.26$ \\
$30-44$ & 284 & 2,220 & 0.69 & $<0.01$ & $0.57-0.83$ \\
$45+$ & 196 & 1,684 & 0.57 & $<0.01$ & $0.46-0.70$ \\
Not answered & 14 & 118 & 0.58 & 0.06 & $0.33-1.02$ \\
Education & & & & & \\
$\leq 7$ years & 136 & 843 & 1.00 & & \\
8-11 years & 511 & 3,339 & 0.99 & 0.92 & $0.79-1.24$ \\
High school & 138 & 830 & 1.05 & 0.72 & $0.80-1.37$ \\
Post-secondary & 329 & 2,146 & 0.98 & 0.86 & $0.78-1.23$ \\
Not answered & 41 & 394 & 0.65 & 0.03 & $0.44-0.96$ \\
\hline
\end{tabular}

Odds ratios by ever and usual occupation and industry categories are shown in Tables 4 and 5 , respectively. In the ever occupation/industry categories and for most major groups, a number of occupations/industries had significantly elevated ORs. In many instances, the corresponding ORs in the usual occupations/industries categories were either non-significant or had fewer than five cases. Concordance for statistically significantly increased ORs between ever and usual categories at least at significant level $\alpha=0.10$ with a minimum of five cases in each category is shown in italics below.

\subsection{Usual Occupation (Table 4)}

In the usual occupation category, significant excess risk in the major occupation groups (two-digit codes) was only observed at $\alpha=0.10$ for occupations in social sciences and related (SOC 23). Excess risks for minor groups (three-digit codes) were significant at $\alpha=0.05$ for occupations in fabricating, assembling and repairing wood products (SOC 854) and at $\alpha=0.10$ for occupations in other managers and administrators (SOC 113/114), bookkeeping and account-recording (SOC 413), and lodging and other accommodation services (SOC 613). Several unit occupations (four-digit codes) had significantly increased ORs at $\alpha=0.05$ for: insurance sales jobs (SOC 5171), rail transport equipment mechanics and repairers (SOC 8583), brick and stone masons-tile setters (SOC 8782), ship engineering officers (SOC 9153), and at $\alpha=0.10$ for: administrators in teaching and related fields (SOC 1133), financial management (SOC 1135), livestock farm workers (SOC 7183), sawmill sawyers and related (SOC 8231), cabinet and wood furniture makers (SOC 8541), and taxi drivers and chauffeurs (SOC 9173); the ORs were significantly low at $\alpha=0.10$ for sales clerks and salespersons in commodities, not else classified (SOC 5135) and welding and flame cutting (SOC 8335). 
Table 4. Odds Ratios for ever and usual occupations.

\begin{tabular}{|c|c|c|c|c|c|c|c|c|c|c|c|}
\hline \multirow{3}{*}{$\begin{array}{l}\text { Code } \\
11\end{array}$} & \multirow{3}{*}{$\begin{array}{l}\text { Occupation Title } \\
\text { Managerial, Administrative and Related }\end{array}$} & \multicolumn{5}{|c|}{ Ever } & \multicolumn{5}{|c|}{ Usual } \\
\hline & & \multirow{2}{*}{$\begin{aligned} \text { Case } \\
309\end{aligned}$} & \multirow{2}{*}{$\frac{\mathbf{O R}}{1.12}$} & \multirow{2}{*}{$\frac{P \text { value }}{0.163}$} & \multicolumn{2}{|c|}{$95 \% \mathrm{CI}$} & \multirow{2}{*}{$\begin{array}{r}\text { Case } \\
173\end{array}$} & \multirow{2}{*}{$\begin{array}{ll}\mathbf{O R} \\
1.17\end{array}$} & \multirow{2}{*}{$\frac{P \text { value }}{0.122}$} & \multicolumn{2}{|c|}{$95 \%$ CI } \\
\hline & & & & & 0.96 & 1.31 & & & & 0.96 & 1.43 \\
\hline 111 & $\begin{array}{l}\text { Officials and Administrators Unique to } \\
\text { Government }\end{array}$ & 40 & 1.19 & 0.343 & 0.83 & 1.71 & 17 & 1.18 & 0.548 & 0.69 & 2.03 \\
\hline 1113 & Administrators & 11 & 1.35 & 0.390 & 0.68 & 2.68 & 5 & 1.37 & 0.539 & 0.50 & 3.74 \\
\hline 1116 & Inspectors and Regulatory Officers & 21 & 1.17 & 0.524 & 0.72 & 1.90 & 11 & 1.33 & 0.402 & 0.68 & 2.59 \\
\hline $113 / 114$ & Other Managers and Administrators & 157 & 1.13 & 0.218 & 0.93 & 1.37 & 84 & 1.24 & 0.099 & 0.97 & 1.58 \\
\hline 1130 & General Managers and Other Senior Officials & 62 & 1.26 & 0.130 & 0.93 & 1.70 & 28 & 1.30 & 0.226 & 0.85 & 1.99 \\
\hline 1131 & Management, Natural Sciences Engineering & 7 & 0.75 & 0.486 & 0.33 & 1.69 & 6 & 1.27 & 0.616 & 0.50 & 3.23 \\
\hline 1133 & Administrators in Teaching and Related Fields & 17 & 1.32 & 0.325 & 0.76 & 2.29 & 10 & 1.96 & 0.076 & 0.93 & 4.12 \\
\hline 1135 & Financial Management & 27 & 1.33 & 0.190 & 0.87 & 2.04 & 17 & 1.59 & 0.099 & 0.93 & 2.73 \\
\hline 1137 & Sales and Advertising Management & 54 & 0.92 & 0.597 & 0.68 & 1.25 & 20 & 0.79 & 0.331 & 0.49 & 1.29 \\
\hline 1143 & Production Management & 31 & 0.94 & 0.764 & 0.63 & 1.41 & 11 & 0.96 & 0.904 & 0.49 & 1.86 \\
\hline 1145 & Management, Construction Operations & 9 & 0.57 & 0.109 & 0.29 & 1.13 & 6 & 0.93 & 0.873 & 0.38 & 2.27 \\
\hline 1147 & $\begin{array}{l}\text { Management, Transport, Communication } \\
\text { Operations }\end{array}$ & 25 & 1.43 & 0.120 & 0.91 & 2.24 & 9 & 1.05 & 0.895 & 0.51 & 2.17 \\
\hline 1149 & Other Managers and Administrators, NEC & 48 & 1.26 & 0.178 & 0.90 & 1.76 & 17 & 1.41 & 0.208 & 0.83 & 2.41 \\
\hline 117 & $\begin{array}{l}\text { Occupations Related to } \\
\text { Management/Administration }\end{array}$ & 68 & 1.10 & 0.504 & 0.83 & 1.46 & 25 & 0.87 & 0.538 & 0.56 & 1.36 \\
\hline 1171 & $\begin{array}{l}\text { Accountants, Auditors and Other Financial } \\
\text { Officers }\end{array}$ & 46 & 1.15 & 0.410 & 0.83 & 1.60 & 23 & 1.02 & 0.934 & 0.64 & 1.63 \\
\hline 21 & $\begin{array}{l}\text { Natural Sciences, Engineering and } \\
\text { Mathematics }\end{array}$ & 84 & 0.94 & 0.632 & 0.73 & 1.21 & 35 & 0.79 & 0.210 & 0.55 & 1.14 \\
\hline $214 / 215$ & $\begin{array}{l}\text { Architects, Engineers and Community } \\
\text { Planners }\end{array}$ & 25 & 0.90 & 0.642 & 0.58 & 1.40 & 13 & 0.95 & 0.869 & 0.52 & 1.75 \\
\hline 216 & $\begin{array}{l}\text { Other Occupations in Architecture and } \\
\text { Engineering }\end{array}$ & 40 & 1.11 & 0.555 & 0.79 & 1.57 & 11 & 0.91 & 0.781 & 0.47 & 1.77 \\
\hline 23 & Social Sciences and Related & 19 & 1.23 & 0.429 & 0.74 & 2.05 & 14 & 1.74 & 0.077 & 0.94 & 3.21 \\
\hline
\end{tabular}


Table 4. Cont

\begin{tabular}{|c|c|c|c|c|c|c|c|c|c|c|c|}
\hline \multirow{3}{*}{$\begin{array}{l}\text { Code } \\
234\end{array}$} & \multirow{3}{*}{$\begin{array}{l}\text { Occupation Title } \\
\text { Law and Jurisprudence }\end{array}$} & \multicolumn{5}{|c|}{ Ever } & \multicolumn{5}{|c|}{ Usual } \\
\hline & & \multirow{2}{*}{$\begin{aligned} \text { Case } \\
10\end{aligned}$} & \multirow{2}{*}{$\frac{\mathbf{O R}}{1.47}$} & \multirow{2}{*}{$\begin{array}{r}P \text { value } \\
0.298\end{array}$} & \multicolumn{2}{|c|}{$95 \% \mathrm{CI}$} & \multirow{2}{*}{$\begin{array}{r}\text { Case } \\
8\end{array}$} & \multirow{2}{*}{$\begin{array}{ll}\text { OR } \\
1.56\end{array}$} & \multirow{2}{*}{$\begin{array}{r}P \text { value } \\
0.282\end{array}$} & \multicolumn{2}{|c|}{$95 \% \mathrm{CI}$} \\
\hline & & & & & 0.71 & 3.04 & & & & 0.69 & 3.51 \\
\hline 2343 & Lawyers and Notaries & 10 & 1.88 & 0.095 & 0.90 & 3.94 & 7 & 1.72 & 0.221 & 0.72 & 4.10 \\
\hline 25 & Religion & 11 & 1.05 & 0.886 & 0.54 & 2.05 & 9 & 1.30 & 0.505 & 0.60 & 2.81 \\
\hline 2511 & Minist & 11 & 1.10 & 0.785 & 0.56 & 2.18 & 9 & 1.30 & 0.505 & 0.60 & 2.81 \\
\hline 27 & Teaching and Related & 52 & 0.88 & 0.441 & 0.64 & 1.22 & 24 & 0.79 & 0.308 & 0.50 & 1.24 \\
\hline 271 & University Teaching an & 12 & 1.05 & 0.879 & 0.56 & 1.97 & 8 & 1.08 & 0.847 & 0.49 & 2.36 \\
\hline 2711 & University Teachers & 10 & 0.99 & 0.977 & 0.50 & 1.95 & 8 & 1.18 & 0.680 & 0.54 & 2.59 \\
\hline 273 & Elementary and Secon & 28 & 0.78 & 0.242 & 0.51 & 1.18 & 13 & 0.70 & 0.251 & 0.38 & 1.29 \\
\hline 2733 & Secondary S & 14 & 0.81 & 0.473 & 0.46 & 1.44 & 6 & 0.69 & 0.408 & 0.29 & 1.66 \\
\hline 31 & Medicine and Health & 31 & 0.81 & 0.299 & 0.54 & 1.21 & 22 & 0.83 & 0.436 & 0.52 & 1.33 \\
\hline 311 & Healtl & 16 & 1.19 & 0.535 & 0.69 & 2.06 & 16 & 1.24 & 0.458 & 0.70 & 2,19 \\
\hline 3111 & ns and Surgeons & 12 & 1.41 & 0.297 & 0.74 & 2.69 & 12 & 1.19 & 0.286 & 0.86 & 1.64 \\
\hline 33 & Artist & 36 & 1.02 & 0.916 & 0.71 & 1.47 & 15 & 1.36 & 0.294 & 0.77 & 2.42 \\
\hline 331 & $\begin{array}{l}\text { Fine and Commercial Art, Photography and } \\
\text { Related }\end{array}$ & 9 & 0.80 & 0.539 & 0.39 & 1.63 & 8 & 1.53 & 0.290 & 0.70 & 3.36 \\
\hline 41 & Cleri & 195 & 1.00 & 0.912 & 0.85 & 1.21 & 54 & 0.99 & 0.948 & 0.73 & 1.34 \\
\hline 413 & Bookke & 65 & 1.08 & 0.597 & 0.81 & 1.44 & 20 & 1.66 & 0.052 & 1.00 & 2.77 \\
\hline 4130 & Supervis & 10 & 1.09 & 0.807 & 0.55 & 2.18 & 5 & 2.33 & 0.125 & 0.79 & 6.87 \\
\hline 4131 & Bookkeepers a & 42 & 1.19 & 0.325 & 0.84 & 1.68 & 5 & 0.86 & 0.761 & 0.33 & 2.28 \\
\hline 415 & $\begin{array}{l}\text { Material Recording, Scheduling and } \\
\text { Distributing }\end{array}$ & 61 & 0.78 & 0.075 & 0.59 & 0.03 & 11 & 0.67 & 0.223 & 0.35 & 1.28 \\
\hline 4155 & Stock Clerks & 22 & 0.64 & 0.065 & 0.40 & 1.03 & 6 & 0.95 & 0.910 & 0.39 & 2.31 \\
\hline 417 & $\begin{array}{l}\text { Reception, Information, Mail, Message } \\
\text { Distribution }\end{array}$ & 28 & 0.90 & 0.611 & 0.60 & 1.35 & 8 & 0.72 & 0.398 & 0.34 & 1.54 \\
\hline 419 & Other Clerical and Related & 73 & 1.25 & 0.100 & 1.00 & 1.61 & 13 & 0.97 & 0.920 & 0.54 & 1.75 \\
\hline 51 & Sales & 279 & 1.09 & 0.264 & 0.94 & 1.27 & 104 & 0.94 & 0.585 & 0.75 & 1.17 \\
\hline $513 / 514$ & Sales, Commodities & 196 & 0.98 & 0.815 & 0.83 & 1.16 & 70 & 0.81 & 0.115 & 0.62 & 1.05 \\
\hline
\end{tabular}


Table 4. Cont

\begin{tabular}{|c|c|c|c|c|c|c|c|c|c|c|c|}
\hline \multirow{3}{*}{$\begin{array}{l}\text { Code } \\
5130\end{array}$} & \multirow{3}{*}{$\begin{array}{l}\text { Occupation Title } \\
\text { Supervisors }\end{array}$} & \multicolumn{5}{|c|}{ Ever } & \multicolumn{5}{|c|}{ Usual } \\
\hline & & \multirow{2}{*}{$\begin{array}{r}\text { Case } \\
85\end{array}$} & \multirow{2}{*}{$\begin{array}{c}\mathbf{O R} \\
0.85\end{array}$} & \multirow{2}{*}{$\begin{array}{r}P \text { value } \\
0.169\end{array}$} & \multicolumn{2}{|c|}{$95 \%$ CI } & \multirow{2}{*}{$\frac{\text { Case }}{35}$} & \multirow{2}{*}{$\begin{array}{ll}\text { OR } \\
0.85\end{array}$} & \multirow{2}{*}{$\begin{array}{r}P \text { value } \\
0.397\end{array}$} & \multicolumn{2}{|c|}{$95 \%$ CI } \\
\hline & & & & & 0.67 & 1.07 & & & & 0.58 & 1.24 \\
\hline 5133 & Commercial Travellers & 49 & 1.25 & 0.181 & 0.90 & 1.73 & 15 & 1.30 & 0.374 & 0.73 & 2.32 \\
\hline 5135 & $\begin{array}{l}\text { Sales Clerks and Salespersons, Commodities, } \\
\text { NEC }\end{array}$ & 89 & 0.86 & 0.196 & 0.68 & 1.08 & 17 & 0.64 & 0.099 & 0.38 & 1.09 \\
\hline 517 & Sales, Services & 72 & 1.39 & 0.021 & 1.05 & 1.84 & 24 & 1.29 & 0.273 & 0.82 & 2.04 \\
\hline 5171 & Insurance Sales & 31 & 1.81 & 0.006 & 1.19 & 2.76 & 9 & 2.22 & 0.043 & 1.02 & 4.81 \\
\hline 5172 & Real Estate Sales & 26 & 1.13 & 0.574 & 0.74 & 1.73 & 9 & 1.65 & 0.190 & 0.78 & 3.49 \\
\hline 519 & Other Sales & 26 & 0.88 & 0.548 & 0.58 & 1.34 & 8 & 1.19 & 0.669 & 0.54 & 2.64 \\
\hline 5193 & Route Drivers & 20 & 1.02 & 0.936 & 0.63 & 1.65 & 6 & 1.21 & 0.684 & 0.48 & 3.03 \\
\hline 61 & Services & 365 & 0.96 & 0.581 & 0.83 & 1.11 & 79 & 0.95 & 0.688 & 0.74 & 1.22 \\
\hline 611 & Protective Services & 260 & 0.98 & 0.815 & 0.83 & 1.16 & 35 & 0.89 & 0.542 & 0.61 & 1.29 \\
\hline 6111 & Firefighters & 9 & 0.95 & 0.889 & 0.40 & 2.25 & 7 & 1.14 & 0.756 & 0.50 & 2.60 \\
\hline 6112 & Police Officers and Detectives, Government & 16 & 1.03 & 0.913 & 0.61 & 1.75 & 6 & 0.79 & 0.596 & 0.33 & 1.89 \\
\hline 6116 & Commissioned Officers, Armed Forces & 44 & 0.88 & 0.465 & 0.63 & 1.24 & 8 & 0.94 & 0.876 & 0.43 & 2.04 \\
\hline 6117 & Other Ranks, Armed Forces & 195 & 1.06 & 0.530 & 0.88 & 1.27 & 7 & 0.76 & 0.499 & 0.349 & 1.68 \\
\hline 612 & Food and Beverage Preparation and Related & 55 & 0.91 & 0.532 & 0.68 & 1.22 & 11 & 0.67 & 0.202 & 0.36 & 1.24 \\
\hline 6121 & Chefs and Cooks & 24 & 1.06 & 0.797 & 0.68 & 1.65 & 5 & 0.70 & 0.454 & 0.28 & 1.78 \\
\hline 613 & Lodging and Other Accommodation Services & 28 & 0.82 & 0.346 & 0.54 & 1.24 & 8 & 2.00 & 0.099 & 0.89 & 4.56 \\
\hline 6130 & Supervisors & 23 & 0.81 & 0.371 & 0.51 & 1.29 & 8 & 1.90 & 0.128 & 0.83 & 4.34 \\
\hline 619 & Other Service & 63 & 1.12 & 0.440 & 0.84 & 1.49 & 18 & 1.09 & 0.749 & 0.64 & 1.85 \\
\hline 6190 & Supervisors & 13 & 2.01 & 0.037 & 1.04 & 3.87 & 5 & 2.05 & 0.180 & 0.72 & 5.85 \\
\hline 6191 & Janitors, Charworkers and Cleaners & 46 & 1.09 & 0.603 & 0.79 & 1.51 & 11 & 0.81 & 0.524 & 0.42 & 1.55 \\
\hline 71 & Farming, Horticultural and Animal Husbandry & 308 & 0.92 & 0.282 & 0.79 & 1.07 & 95 & 1.06 & 0.647 & 0.83 & 1.36 \\
\hline 711 & Farmers & 182 & 1.00 & 0.919 & 0.83 & 1.22 & 79 & 1.13 & 0.353 & 0.87 & 1.46 \\
\hline 7113 & Livestock Farmers & 46 & 1.22 & 0.247 & 0.87 & 1.71 & 19 & 1.30 & 0.323 & 0.77 & 2.19 \\
\hline 7115 & Crop Farmers & 46 & 1.09 & 0.619 & 0.78 & 1.53 & 16 & 0.96 & 0.886 & 0.55 & 1.68 \\
\hline
\end{tabular}


Table 4. Cont.

\begin{tabular}{|c|c|c|c|c|c|c|c|c|c|c|c|}
\hline \multirow{3}{*}{$\begin{array}{l}\text { Code } \\
718 / 719\end{array}$} & \multirow{3}{*}{$\begin{array}{l}\text { Occupation Title } \\
\text { Other Farming, Horticulture and Animal } \\
\text { Husbandry }\end{array}$} & \multicolumn{5}{|c|}{ Ever } & \multicolumn{5}{|c|}{ Usual } \\
\hline & & \multirow{2}{*}{$\begin{array}{r}\text { Case } \\
63\end{array}$} & \multirow{2}{*}{$\frac{\text { OR }}{0.84}$} & \multirow{2}{*}{$\frac{\boldsymbol{P} \text { value }}{0.234}$} & \multicolumn{2}{|c|}{$95 \%$ CI } & \multirow{2}{*}{$\begin{array}{r}\text { Case } \\
6\end{array}$} & \multirow{2}{*}{$\begin{array}{l}\text { OR } \\
1.02\end{array}$} & \multirow{2}{*}{$\begin{array}{r}P \text { value } \\
0.965\end{array}$} & \multicolumn{2}{|c|}{$95 \% \mathrm{CI}$} \\
\hline & & & & & 0.63 & 1.12 & & & & 0.43 & 2.44 \\
\hline 7183 & Livestock Farm Workers & 31 & 1.00 & 0.961 & 0.68 & 1.51 & 5 & 2.58 & 0.077 & 0.90 & 7.36 \\
\hline 7195 & Nursery and Related Workers & 21 & 1.19 & 0.485 & 0.73 & 1.94 & 6 & 1.47 & 0.420 & 0.58 & 3.75 \\
\hline 7199 & $\begin{array}{l}\text { Other Farming, Horticulture and Animal } \\
\text { Husbandry, NEC }\end{array}$ & 80 & 0.86 & 0.228 & 0.67 & 1.10 & 7 & 0.88 & 0.754 & 0.40 & 1.96 \\
\hline 73 & Fishing, Trapping and Related & 47 & 1.20 & 0.278 & 0.86 & 1.67 & 13 & 0.93 & 0.814 & 0.51 & 1.70 \\
\hline 7313 & Net, Trap and Line Fishing & 44 & 1.25 & 0.198 & 0.89 & 1.76 & 12 & 0.91 & 0.766 & 0.49 & 1.70 \\
\hline 75 & Forestry and Logging & 121 & 1.01 & 0.924 & 0.82 & 1.24 & 31 & 1.30 & 0.198 & 0.87 & 1.94 \\
\hline 7510 & Foremen/women & 21 & 1.09 & 0.724 & 0.68 & 1.76 & 6 & 1.14 & 0.778 & 0.46 & 2.84 \\
\hline 7513 & Timber Cutting and Related & 64 & 1.07 & 0.646 & 0.80 & 1.43 & 13 & 1.39 & 0.294 & 0.75 & 2.57 \\
\hline 77 & Mining and Quarrying & 71 & 0.88 & 0.327 & 0.68 & 1.14 & 16 & 1.36 & 0.294 & 0.77 & 2.42 \\
\hline 7717 & Mineral Cutting, Handling and Loading & 34 & 0.79 & 0.235 & 0.54 & 1.17 & 8 & 1.40 & 0.405 & 0.63 & 3.09 \\
\hline $81 / 82$ & Materials Processing & 54 & 0.78 & 0.096 & 0.58 & 1.05 & 74 & 1.02 & 0.881 & 0.79 & 1.32 \\
\hline $813 / 814$ & Metal Processing and Related & 18 & 0.82 & 0.435 & 0.50 & 1.35 & 5 & 0.96 & 0.933 & 0.37 & 2.50 \\
\hline $821 / 822$ & Food, Beverage and Related Processing & 47 & 1.31 & 0.113 & 0.94 & 1.83 & 12 & 1.03 & 0.926 & 0.55 & 1.92 \\
\hline 8215 & $\begin{array}{l}\text { Slaughtering, Meat Cutting, Canning, Curing, } \\
\text { Packing }\end{array}$ & 25 & 1.29 & 0.259 & 0.83 & 2.01 & 11 & 1.55 & 0.221 & 0.77 & 3.13 \\
\hline 823 & $\begin{array}{l}\text { Wood Processing (Non-Pulp and Paper } \\
\text { Production) }\end{array}$ & 132 & 1.13 & 0.247 & 0.92 & 1.39 & 41 & 1.28 & 0.173 & 0.90 & 1.83 \\
\hline 8231 & Sawmill Sawyers and Related & 43 & 1.19 & 0.306 & 0.85 & 1.66 & 16 & 1.77 & 0.053 & 0.99 & 3.15 \\
\hline 8238 & Labouring & 51 & 1.11 & 0.520 & 0.81 & 1.53 & 7 & 0.93 & 0.861 & 0.41 & 2.10 \\
\hline 8239 & Wood Processing, Non-Pulp and Paper, NEC & 40 & 1.23 & 0.241 & 0.87 & 1.74 & 8 & 1.50 & 0.327 & 0.67 & 3.37 \\
\hline 825 & Pulp and Paper Production and Related & 21 & 0.85 & 0.504 & 0.53 & 1.37 & 5 & 0.74 & 0.524 & 0.29 & 1.87 \\
\hline 83 & Machining and Related & 117 & 1.05 & 0.650 & 0.85 & 1.30 & 31 & 0.73 & 0.106 & 0.50 & 1.07 \\
\hline 831 & Metal Machining & 51 & 1.27 & 0.145 & 0.92 & 1.75 & 15 & 1.09 & 0.771 & 0.61 & 1.95 \\
\hline 8313 & Machinist and Machine Tool Setting-up & 37 & 1.14 & 0.497 & 0.78 & 1.66 & 6 & 0.58 & 0.219 & 0.24 & 1.38 \\
\hline
\end{tabular}


Table 4. Cont.

\begin{tabular}{|c|c|c|c|c|c|c|c|c|c|c|c|}
\hline \multirow{3}{*}{$\begin{array}{l}\text { Code } \\
833\end{array}$} & \multirow{3}{*}{$\begin{array}{l}\text { Occupation Title } \\
\text { Metal Shaping and Forming, Except } \\
\text { Machining }\end{array}$} & \multicolumn{5}{|c|}{ Ever } & \multicolumn{5}{|c|}{ Usual } \\
\hline & & \multirow{2}{*}{$\begin{array}{r}\text { Case } \\
62\end{array}$} & \multirow{2}{*}{$\begin{array}{l}\text { OR } \\
1.08\end{array}$} & \multirow{2}{*}{$\frac{P \text { value }}{0.597}$} & \multicolumn{2}{|c|}{$95 \% \mathrm{CI}$} & \multirow{2}{*}{$\begin{array}{r}\text { Case } \\
15\end{array}$} & \multirow{2}{*}{$\frac{\text { OR }}{0.71}$} & \multirow{2}{*}{$\begin{array}{r}P \text { value } \\
0.217\end{array}$} & \multicolumn{2}{|c|}{ 95\% CI } \\
\hline & & & & & 0.81 & 1.44 & & & & 0.41 & 1.22 \\
\hline 8335 & Welding and Flame Cutting & 36 & 0.99 & 0.957 & 0.69 & 1.42 & 7 & 0.49 & 0.081 & 0.22 & 1.09 \\
\hline 85 & $\begin{array}{l}\text { Product Fabricating, Assembling and } \\
\text { Repairing }\end{array}$ & 274 & 1.05 & 0.508 & 0.91 & 1.21 & 114 & 1.08 & 0.487 & 0.87 & 1.34 \\
\hline 853 & Electrical, Electronic Equipment & 56 & 1.36 & 0.506 & 1.00 & 1.85 & 13 & 0.69 & 0.219 & 0.38 & 1.25 \\
\hline 8533 & $\begin{array}{l}\text { Electrical Equipment Installing, Repairing, } \\
\text { NEC }\end{array}$ & 33 & 1.38 & 0.111 & 0.93 & 2.05 & 8 & 0.80 & 0.554 & 0.38 & 1.68 \\
\hline 854 & $\begin{array}{l}\text { Fabricating, Assembling and Repairing: Wood } \\
\text { Products }\end{array}$ & 23 & 1.30 & 0.269 & 0.82 & 2.07 & 9 & 2.29 & 0.045 & 1.02 & 5.15 \\
\hline 8541 & Cabinet and Wood Furniture Makers & 15 & 1.48 & 0.195 & 0.82 & 2.68 & 6 & 2.51 & 0.063 & 0.95 & 6.64 \\
\hline 858 & Mechanics and Repairers, NEC & 159 & 1.10 & 0.319 & 0.91 & 1.33 & 69 & 1.11 & 0.437 & 0.85 & 1.44 \\
\hline 8581 & Motor Vehicle Mechanics and Repairers & 63 & 1.08 & 0.597 & 0.81 & 1.44 & 20 & 0.87 & 0.572 & 0.54 & 1.41 \\
\hline 8582 & Aircraft Mechanics and Repairers & 24 & 1.35 & 0.211 & 0.84 & 2.16 & 5 & 1.15 & 0.783 & 0.43 & 3.10 \\
\hline 8583 & $\begin{array}{l}\text { Rail Transport Equipment Mechanics and } \\
\text { Repairers }\end{array}$ & 11 & 3.84 & $<0.001$ & 1.82 & 8.11 & 6 & 6.06 & 0.001 & 2.04 & 18.00 \\
\hline 8584 & Other Industrial Equipment Repairers & 64 & 0.96 & 0.774 & 0.73 & 1.27 & 28 & 1.03 & 0.888 & 0.68 & 1.55 \\
\hline 8589 & Other Mechanics and Repairers, NEC & 13 & 0.98 & 0.948 & 0.54 & 1.79 & 6 & 2.02 & 0.147 & 0.78 & 5.22 \\
\hline 859 & $\begin{array}{l}\text { Other Product Fabrication, Assembling, } \\
\text { Repairing }\end{array}$ & 49 & 0.93 & 0.658 & 0.67 & 1.28 & 11 & 1.17 & 0.643 & 0.60 & 2.27 \\
\hline 8592 & $\begin{array}{l}\text { Marine Craft Fabricating, Assembling, } \\
\text { Repairing }\end{array}$ & 36 & 1.01 & 0.958 & 0.70 & 1.46 & 9 & 1.65 & 0.198 & 0.77 & 3.54 \\
\hline 87 & Construction Trades & 273 & 0.90 & 0.184 & 0.77 & 1.05 & 110 & 0.85 & 0.137 & 0.69 & 1.05 \\
\hline 871 & Excavating, Grading, Paving & 63 & 0.94 & 0.671 & 0.71 & 1.25 & 15 & 0.66 & 0.130 & 0.39 & 1.13 \\
\hline 8711 & Excavating, Grading and Related & 31 & 1.02 & 0.922 & 0.69 & 1.52 & 9 & 0.80 & 0.539 & 0.39 & 1.63 \\
\hline 873 & $\begin{array}{l}\text { Electrical, Wire Communications } \\
\text { Installing/Repair }\end{array}$ & 42 & 1.03 & 0.867 & 0.73 & 1.46 & 18 & 1.00 & 0.999 & 0.60 & 1.71 \\
\hline
\end{tabular}


Table 4. Cont

\begin{tabular}{|c|c|c|c|c|c|c|c|c|c|c|c|}
\hline \multirow{3}{*}{$\begin{array}{l}\text { Code } \\
8733\end{array}$} & \multirow{3}{*}{$\begin{array}{l}\text { Occupation Title } \\
\text { Electricians and Repairers }\end{array}$} & \multicolumn{5}{|c|}{ Ever } & \multicolumn{5}{|c|}{ Usual } \\
\hline & & \multirow{2}{*}{$\begin{array}{r}\text { Case } \\
24\end{array}$} & \multirow{2}{*}{$\begin{array}{l}\text { OR } \\
1.07\end{array}$} & \multirow{2}{*}{$\begin{array}{c}\boldsymbol{P} \text { value } \\
0.771\end{array}$} & \multicolumn{2}{|c|}{$95 \% \mathrm{CI}$} & \multirow{2}{*}{$\begin{array}{r}\text { Case } \\
12\end{array}$} & \multirow{2}{*}{$\begin{array}{l}\text { OR } \\
1.37\end{array}$} & \multirow{2}{*}{$\begin{array}{r}\boldsymbol{P} \text { value } \\
0.336\end{array}$} & \multicolumn{2}{|c|}{$95 \%$ CI } \\
\hline & & & & & 0.68 & 1.69 & & & & 0.72 & 2.60 \\
\hline $878 / 879$ & Other Construction Trade & 112 & 0.84 & 0.116 & 0.68 & 1.04 & 50 & 0.85 & 319 & 0.62 & 1.17 \\
\hline 8780 & Foremer & 20 & 0.92 & & 0.58 & 1.47 & 6 & 0.96 & 27 & 0.40 & 2.31 \\
\hline & $\mathrm{C}$ & 70 & 0.86 & & 0.66 & 1.12 & 27 & 0.80 & 03 & 0.52 & 1.22 \\
\hline 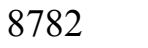 & B & 9 & 1.29 & 0.493 & 0.62 & 2.67 & 7 & 2.97 & & 1.20 & 7.34 \\
\hline & $\mathrm{P}$ & 27 & 0.99 & & 0.64 & 1.52 & 17 & 1.20 & & 0.71 & 2.04 \\
\hline & $\mathrm{L}$ & 44 & 0.94 & 17 & 0.67 & 1. & 5 & 0.75 & & 0.29 & 1.93 \\
\hline Sו & $\mathrm{C}$ & 10 & 0.80 & 0.507 & 0.41 & 1. & 5 & 1.79 & 56 & 0.66 & 4.89 \\
\hline 91 & & 246 & 1.15 & 0.077 & 0.99 & 1.3 & 86 & 1.15 & 79 & 0.89 & 1.48 \\
\hline 91 & A & 24 & 1.51 & 0.084 & 0.95 & 2.4 & 10 & 1.39 & 359 & 0.69 & 2.81 \\
\hline & & 16 & 1.29 & 0.368 & 0.74 & 2.2 & 7 & 1.24 & 618 & 0.53 & 2.89 \\
\hline & & 23 & 1.14 & & 0.73 & 1.7 & 12 & 1.29 & & 0.68 & 2.46 \\
\hline & & 8 & 0.71 & & 0.33 & 1.5 & 6 & 1.4 & & 0.55 & 3.56 \\
\hline & & 13 & 1.79 & & 0.95 & 3. & 6 & 2.05 & & 0.78 & 5.40 \\
\hline & & 51 & 1.17 & & 0.86 & 1.60 & 16 & 1.17 & & 0.68 & 2.02 \\
\hline & & 13 & 0.88 & & 0.48 & 1. & 6 & 0.94 & & 0.39 & 2.26 \\
\hline & & 10 & 1.56 & & 0.77 & 3.17 & 7 & 4.80 & 02 & 1.81 & 12.71 \\
\hline 91 & $\mathrm{M}$ & 157 & 1.10 & & 0.91 & 1. & 46 & 1.01 & 52 & 0.73 & 40 \\
\hline & & 0 & 0.84 & & 0.52 & 1. & 7 & 0.80 & & 0.36 & 1.77 \\
\hline & & 0 & 1.54 & & 1.01 & & 6 & 2.2 & & 0.89 & 5.71 \\
\hline & & 124 & 1.08 & & 0.88 & 1. & 32 & 0.93 & 16 & 0.63 & 1.3 \\
\hline 93 & & 101 & 1.04 & 4 & 0.83 & 1.3 & 26 & 0.99 & 0.963 & 0.64 & 1.52 \\
\hline 931 & nd Freight & 48 & 1.13 & 0.462 & 0.82 & 1.5 & 16 & 1.33 & 0.320 & 0.76 & 2.33 \\
\hline & & & 0.93 & & 0.59 & & 5 & & & 0.37 & 2.39 \\
\hline 95 & & 68 & 1.11 & 0.4 & 0.84 & 1.46 & 29 & 1.02 & 0.925 & 0.67 & 1.55 \\
\hline 953 & $\begin{array}{l}\text { Stationary Engine, Utilities Equipment } \\
\text { Operating }\end{array}$ & 37 & 1.16 & 0.432 & $\begin{array}{r}0.80 \\
5\end{array}$ & 1.68 & 16 & 1.22 & 0.477 & 0.71 & 2.11 \\
\hline 953 & Statio & 25 & 1.03 & 0.892 & 0.67 & 1.58 & 12 & 1.18 & 0.612 & 0.62 & 2.24 \\
\hline 955 & Electronic Communications Equipment, NEC & 19 & 1.46 & 0.158 & 0.86 & 2.47 & 9 & 1.75 & 0.159 & 0.80 & 3.82 \\
\hline
\end{tabular}


Table 5. Odds Ratios for ever and usual industries.

\begin{tabular}{|c|c|c|c|c|c|c|c|c|c|c|c|}
\hline \multirow{3}{*}{$\begin{array}{l}\text { Code } \\
01\end{array}$} & \multirow{3}{*}{$\begin{array}{l}\text { Industry Title } \\
\text { Agriculture }\end{array}$} & \multicolumn{5}{|c|}{ Ever } & \multicolumn{5}{|c|}{ Usual } \\
\hline & & \multirow{2}{*}{$\begin{aligned} \text { Case } \\
296\end{aligned}$} & OR & \multirow{2}{*}{$\frac{P \text { value }}{0.184}$} & \multicolumn{2}{|c|}{$95 \% \mathrm{CI}$} & \multicolumn{2}{|c|}{ Case OR } & \multirow{2}{*}{$\begin{array}{r}P \text { value } \\
0.999\end{array}$} & \multicolumn{2}{|c|}{$95 \%$ CI } \\
\hline & & & 0.90 & & 0.77 & 1.05 & 87 & 1.00 & & 0.78 & 1.31 \\
\hline 011 & Livestock Farms (except Animal Specialities) & 72 & 1.13 & 0.377 & 0.86 & 1.48 & 22 & 1.53 & 0.085 & 0.94 & 2.48 \\
\hline 0111 & Dairy Farms & 49 & 1.39 & 0.046 & 1.01 & 1.92 & 8 & 1.13 & 0.757 & 0.52 & 2.45 \\
\hline 0112 & Cattle Farms & 13 & 0.88 & 0.678 & 0.48 & 1.61 & 6 & 1.33 & 0.556 & 0.52 & 3.43 \\
\hline 0114 & Poultry and Egg Farms & 17 & 1.56 & 0.116 & 0.90 & 2.72 & 6 & 3.52 & 0.016 & 1.26 & 9.81 \\
\hline 013 & Field Crop Farms & 49 & 0.96 & 0.806 & 0.69 & 1.33 & 9 & 0.98 & 0.958 & 0.46 & 2.09 \\
\hline 0131 & Wheat Farms & 34 & 0.95 & 0.792 & 0.65 & 1.39 & 6 & 0.92 & 0.861 & 0.36 & 2.34 \\
\hline 015 & Fruit and Other Vegetable Farms & 35 & 1.04 & 0.837 & 0.72 & 1.51 & 9 & 0.95 & 0.889 & 0.46 & 1.95 \\
\hline 0151 & Fruit Farms & 32 & 1.04 & 0.844 & 0.71 & 1.54 & 9 & 1.07 & 0.856 & 0.52 & 2.22 \\
\hline 017 & $\begin{array}{l}\text { Livestock, Field Crop, Horticultural Combination } \\
\text { Farms }\end{array}$ & 179 & 0.87 & 0.157 & 0.72 & 1.06 & 45 & 0.93 & 0.674 & 0.66 & 1.31 \\
\hline 03 & Fishing and Trapping & 49 & 1.08 & 0.631 & 0.79 & 1.48 & 14 & 0.90 & 0.725 & 0.50 & 1.62 \\
\hline 031 & Fishing & 46 & 1.15 & 0.410 & 0.83 & 1.60 & 13 & 0.91 & 0.758 & 0.50 & 1.66 \\
\hline 0311 & Salt Water Fishing & 42 & 1.14 & 0.463 & 0.80 & 1.61 & 12 & 0.87 & 0.656 & 0.47 & 1.61 \\
\hline 04 & Logging & 135 & 0.99 & 0.920 & 0.81 & 1.20 & 38 & 1.13 & 0.515 & 0.78 & 1.63 \\
\hline 06 & Mining & 95 & 0.87 & 0.260 & 0.68 & 1.11 & 21 & 0.89 & 0.627 & 0.56 & 1.42 \\
\hline 061 & Metal Mines & 71 & 0.83 & 0.181 & 0.63 & 1.09 & 17 & 0.90 & 0.693 & 0.53 & 1.52 \\
\hline 0614 & Silver-Lead-Zinc Mines & 23 & 1.10 & 0.682 & 0.70 & 1.74 & 5 & 0.95 & 0.920 & 0.35 & 2.59 \\
\hline 0631 & Bituminous Coal Mines & 28 & 1.08 & 0.719 & 0.71 & 1.64 & 5 & 1.25 & 0.662 & 0.46 & 3.40 \\
\hline 10 & Food & 86 & 1.08 & 0.537 & 0.85 & 1.38 & 22 & 0.96 & 0.863 & 0.60 & 1.53 \\
\hline 101 & Meat and Poultry Products & 18 & 0.92 & 0.748 & 0.55 & 1.53 & 5 & 0.73 & 0.514 & 0.28 & 1.88 \\
\hline 1011 & Meat and Meat Products (Except Poultry) & 18 & 1.01 & 0.970 & 0.60 & 1.71 & 5 & 0.77 & 0.599 & 0.29 & 2.04 \\
\hline 104 & Dairy Products & 21 & 1.34 & 0.239 & 0.82 & 2.18 & 8 & 2.02 & 0.091 & 0.90 & 4.56 \\
\hline 1041 & Fluid Milk & 13 & 1.45 & 0.242 & 0.78 & 2.70 & 5 & 2.08 & 0.173 & 0.73 & 5.96 \\
\hline 11 & Beverage & 13 & 1.24 & 0.482 & 0.68 & 2.26 & 6 & 2.25 & 0.084 & 0.90 & 5.64 \\
\hline 113 & Brewery Products & 8 & 1.99 & 0.090 & 0.90 & 4.41 & 5 & 3.71 & 0.015 & 1.29 & 10.66 \\
\hline
\end{tabular}


Table 5. Cont

\begin{tabular}{|c|c|c|c|c|c|c|c|c|c|c|c|}
\hline \multirow{3}{*}{$\begin{array}{l}\text { Code } \\
25\end{array}$} & \multirow{3}{*}{$\begin{array}{l}\text { Industry Title } \\
\text { Wood }\end{array}$} & \multicolumn{5}{|c|}{ Ever } & \multicolumn{5}{|c|}{ Usual } \\
\hline & & \multicolumn{2}{|c|}{ Case OR } & \multirow{2}{*}{$\frac{P \text { value }}{0.461}$} & \multicolumn{2}{|c|}{$95 \% \mathrm{CI}$} & \multicolumn{2}{|c|}{ Case OR } & \multirow{2}{*}{$\begin{array}{r}P \text { value } \\
0.504\end{array}$} & \multicolumn{2}{|c|}{$95 \% \mathrm{CI}$} \\
\hline & & 189 & 1.07 & & 0.89 & 1.28 & 67 & 1.10 & & 0.83 & 1.46 \\
\hline 251 & Sawmill, Planing Mill and Shingle Mill Products & 153 & 1.02 & 0.838 & 0.84 & 1.23 & 56 & 1.15 & 0.370 & 0.85 & 1.56 \\
\hline 2512 & $\begin{array}{l}\text { Sawmill, Planing Mill (Except Shingles and } \\
\text { Shakes) }\end{array}$ & 147 & 1.03 & 0.773 & 0.84 & 1.26 & 54 & 1.18 & 0.295 & 0.87 & 1.61 \\
\hline 252 & Veneer and Plywood & 22 & 1.25 & 0.356 & 0.78 & 2.01 & 9 & 1.18 & 0.656 & 0.57 & 2.45 \\
\hline 2522 & Softwood Veneer and Plywood & 20 & 1.43 & 0.161 & 0.87 & 2.36 & 9 & 1.55 & 0.248 & 0.74 & 3.26 \\
\hline 26 & Furniture and Fixtures & 16 & 1.14 & 0.639 & 0.66 & 1.97 & 6 & 1.52 & 0.366 & 0.61 & 3.77 \\
\hline 261 & Household Furniture & 14 & 1.19 & 0.569 & 0.65 & 2.17 & 5 & 1.46 & 0.450 & 0.55 & 3.907 \\
\hline 27 & Paper and Allied Products & 49 & 0.89 & 0.451 & 0.66 & 1.21 & 19 & 0.81 & 0.393 & 0.50 & 1.31 \\
\hline 271 & Pulp and Paper Production & 45 & 0.87 & 0.407 & 0.63 & 1.21 & 18 & 0.80 & 0.394 & 0.48 & 1.34 \\
\hline 2711 & Pulp Industry & 35 & 0.79 & 0.210 & 0.55 & 1.14 & 16 & 0.87 & 0.617 & 0.50 & 1.50 \\
\hline 28 & Printing, Publishing and Allied & 24 & 0.81 & 0.348 & 0.52 & 1.26 & 8 & 0.52 & 0.082 & 0.25 & 1.09 \\
\hline 281 & Commercial Printing & 10 & 0.93 & 0.832 & 0.48 & 1.82 & 5 & 0.89 & 0.811 & 0.34 & 2.31 \\
\hline 29 & Primary Metal & 48 & 0.77 & 0.106 & 0.56 & 1.06 & 16 & 0.78 & 0.358 & 0.46 & 1.33 \\
\hline 299 & $\begin{array}{l}\text { Other Rolled, Cast, Extruded Non Ferrous Metal } \\
\text { Products }\end{array}$ & 24 & 0.92 & 0.717 & 0.59 & 1.45 & 10 & 0.98 & 0.954 & 0.49 & 1.95 \\
\hline 30 & $\begin{array}{l}\text { Fabricated Metal Products(Non Machinery, } \\
\text { Transport) }\end{array}$ & 69 & 1.13 & 0.377 & 0.86 & 1.48 & 19 & 0.92 & 0.748 & 0.55 & 1.53 \\
\hline 308 & Machine Shops & 28 & 1.49 & 0.068 & 0.97 & 2.29 & 6 & 1.18 & 0.715 & 0.49 & 2.87 \\
\hline 31 & Machinery (except Electrical) & 28 & 1.63 & 0.025 & 1.07 & 2.50 & 8 & 1.57 & 0.262 & 0.71 & 3.46 \\
\hline 319 & Other Machinery and Equipment & 24 & 1.82 & 0.012 & 1.14 & 2.90 & 8 & 1.86 & 0.135 & 0.83 & 4.20 \\
\hline 3192 & $\begin{array}{l}\text { Construction, Mining Machinery, Materials } \\
\text { Handling }\end{array}$ & 7 & 1.88 & 0.146 & 0.80 & 4.41 & 5 & 3.66 & 0.023 & 1.19 & 11.22 \\
\hline 32 & Transportation Equipment & 115 & 0.98 & 0.852 & 0.79 & 1.21 & 19 & 0.93 & 0.777 & 0.56 & 1.54 \\
\hline 327 & Shipbuilding and Repairs & 73 & 0.95 & 0.705 & 0.73 & 1.24 & 12 & 1.01 & 0.975 & 0.54 & 1.88 \\
\hline 33 & Electrical and Electronic Products & 21 & 1.23 & 0.401 & 0.76 & 1.99 & 6 & 0.95 & 0.907 & 0.40 & 2.25 \\
\hline
\end{tabular}


Table 5. Cont.

\begin{tabular}{|c|c|c|c|c|c|c|c|c|c|c|c|}
\hline \multirow{3}{*}{$\begin{array}{l}\text { Code } \\
36\end{array}$} & \multirow{3}{*}{$\begin{array}{l}\text { Industry Title } \\
\text { Refined Petroleum and Coal Products }\end{array}$} & \multicolumn{5}{|c|}{ Ever } & \multicolumn{5}{|c|}{ Usual } \\
\hline & & \multicolumn{2}{|c|}{ Case OR } & \multirow{2}{*}{$\frac{P \text { value }}{0.368}$} & \multicolumn{2}{|c|}{$95 \%$ CI } & \multicolumn{2}{|c|}{ Case OR } & \multirow{2}{*}{\begin{tabular}{r|}
$\boldsymbol{P}$ value \\
0.288
\end{tabular}} & \multicolumn{2}{|c|}{$95 \%$ CI } \\
\hline & & 16 & 1.29 & & 0.74 & 2.24 & 7 & 1.61 & & 0.67 & 3.88 \\
\hline 361 & Refined Petroleum Products & 13 & 1.17 & 0.615 & 0.63 & 2.16 & 7 & 1.61 & 0.297 & 0.66 & 3.94 \\
\hline 1 & $\begin{array}{l}\text { Refined Petroleum Products(Non Lubricating Oil, } \\
\text { Grease) }\end{array}$ & 13 & & & 0.63 & & 7 & 1.61 & & 0.66 & 3.94 \\
\hline 37 & Chemical and Chemical Products & 31 & 1.42 & 0.091 & 0.95 & 2.13 & 10 & 1.15 & 0.695 & 0.57 & 2.31 \\
\hline 40 & Building, & 130 & 0.93 & 0.497 & 0.75 & 1.15 & 42 & 0.94 & 0.717 & 0.67 & 1.31 \\
\hline 01 & Residentia & 29 & 0.93 & 0.728 & 0.62 & 1.40 & 8 & 1.00 & 0.999 & 0.46 & 2.23 \\
\hline 11 & Single I & 26 & 1.00 & 0.964 & 0.65 & 1.56 & 8 & 1.25 & 0.583 & 0.56 & 2.78 \\
\hline 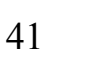 & Indus & 53 & 0.78 & 0.096 & 0.58 & 1.05 & 15 & 0.72 & 0.228 & 0.42 & 1.23 \\
\hline & & 44 & 0.82 & 0.243 & 0.59 & 1.14 & 13 & 0.78 & 0.400 & 0.44 & 1.39 \\
\hline 4121 & Highn & 29 & 0.93 & 0.728 & 0.62 & 1.40 & 9 & 0.99 & 0.978 & 0.48 & 2.39 \\
\hline 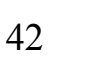 & Trade & 109 & 0.90 & 0.342 & 0.72 & 1.12 & 48 & 0.90 & 0.514 & 0.66 & 1.23 \\
\hline 422 & Stru & 20 & 0.90 & 0.669 & 0.56 & 1.46 & 6 & 0.73 & 0.481 & 0.30 & 1.75 \\
\hline 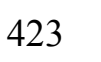 & Ext & 14 & & & 0.66 & & 8 & 1.79 & & 0.81 & 3.96 \\
\hline 231 & Extel & 5 & 1.10 & 0.846 & 0.42 & 2.88 & 5 & 3.02 & 0.045 & 1.03 & 8.89 \\
\hline 424 & Plumbin & 23 & 1.18 & & 0.74 & 1.88 & 12 & 1.07 & 0.834 & 0.57 & 2.01 \\
\hline 4241 & Plumbing & 13 & 0.90 & & 0.49 & 1.65 & 6 & 0.75 & 0.519 & 0.31 & 1.80 \\
\hline 426 & Electrical & 23 & 1.26 & 0.334 & 0.79 & 2.01 & 10 & 1.48 & 0.285 & 0.72 & 3.04 \\
\hline 427 & Interi & 21 & 0.69 & 0.112 & 0.44 & 1.09 & 6 & 0.44 & 0.051 & 0.19 & 1.01 \\
\hline 45 & Trans & 282 & 1.21 & 0.011 & 1.05 & 1.40 & 106 & 1.08 & 0.513 & 0.86 & 1.36 \\
\hline 451 & Air Tra & 24 & 1.26 & 0.320 & 0.80 & 1.99 & 12 & 1.07 & 0.834 & 0.57 & 2.01 \\
\hline 4511 & Schedu & 16 & 0.96 & 0.882 & 0.56 & 1.65 & 10 & 1.03 & 0.933 & 0.52 & 2.04 \\
\hline 453 & Rail Trans & 78 & 1.10 & 0.459 & 0.86 & 1.42 & 35 & 1.17 & 0.419 & 0.80 & 1.71 \\
\hline 4531 & Railway Transport & 77 & 1.10 & 0.459 & 0.86 & 1.42 & 34 & 1.16 & 0.449 & 0.79 & 1.70 \\
\hline 454 & Water Transport & 54 & 1.36 & 0.051 & 1.00 & 1.85 & 9 & 0.70 & 0.316 & 0.35 & 1.41 \\
\hline 4541 & Freight and Passenger Water Transport & 42 & 1.52 & 0.020 & 1.07 & 2.16 & 5 & 0.68 & 0.419 & 0.27 & 1.73 \\
\hline 455 & Services Incidental to Water Transport & 25 & 1.46 & 0.109 & 0.92 & 2.32 & 11 & 1.33 & 0.413 & 0.67 & 2.63 \\
\hline
\end{tabular}


Table 5. Cont.

\begin{tabular}{|c|c|c|c|c|c|c|c|c|c|c|c|}
\hline \multirow{3}{*}{$\begin{array}{l}\text { Code } \\
4551\end{array}$} & \multirow{3}{*}{$\begin{array}{l}\text { Industry Title } \\
\text { Marine Cargo Handling }\end{array}$} & \multicolumn{5}{|c|}{ Ever } & \multicolumn{5}{|c|}{ Usual } \\
\hline & & \multirow{2}{*}{$\begin{aligned} \text { Case } \\
15\end{aligned}$} & \multirow{2}{*}{$\frac{\mathbf{O R}}{1.24}$} & \multirow{2}{*}{$\begin{array}{r}P \text { value } \\
0.458\end{array}$} & \multicolumn{2}{|c|}{ 95\% CI } & \multirow{2}{*}{$\frac{\text { Case }(}{10}$} & \multirow{2}{*}{$\frac{\mathrm{OR}}{1.54}$} & \multirow{2}{*}{$\frac{P \text { value }}{0.232}$} & \multicolumn{2}{|c|}{$95 \%$ CI } \\
\hline & & & & & 0.70 & 2.19 & & & & 0.76 & 3.13 \\
\hline 456 & Truck Transport & 72 & 1.02 & 0.881 & 0.79 & 1.32 & 22 & 0.93 & 0.759 & 0.59 & 1.48 \\
\hline 4561 & General Freight Trucking & 50 & 1.01 & 0.950 & 0.74 & 1.37 & 11 & 0.59 & 0.096 & 0.32 & 1.10 \\
\hline 4562 & Used Goods Moving and Storage & 7 & 1.92 & 0.133 & 0.82 & 4.50 & 5 & 7.14 & 0.002 & 2.112 & 24.13 \\
\hline 457 & Public Passenger Transit Systems & 26 & 0.91 & 0.659 & 0.60 & 1.38 & 8 & 0.83 & 0.629 & 0.39 & 1.77 \\
\hline 4571 & Urban Transit Systems & 16 & 0.82 & 0.474 & 0.48 & 1.41 & 5 & 0.78 & 0.610 & 0.30 & 2.03 \\
\hline 458 & Other Transportation & 28 & 1.58 & 0.038 & 1.03 & 2.43 & 5 & 1.90 & 0.209 & 0.70 & 5.17 \\
\hline 4581 & Taxicab & 26 & 1.52 & 0.070 & 0.97 & 2.39 & 5 & 1.90 & 0.209 & 0.70 & 5.17 \\
\hline 48 & Communications & 54 & 1.06 & 0.709 & 0.78 & 1.44 & 25 & 0.91 & 0.673 & 0.59 & 1.41 \\
\hline 481 & Telecommunication Broadcasting & 9 & 1.30 & 0.486 & 0.62 & 2.72 & 5 & 1.55 & 0.398 & 0.56 & 4.29 \\
\hline 4821 & Telecommunication Carriers & 26 & 1.33 & 0.203 & 0.86 & 2.06 & 13 & 1.16 & 0.629 & 0.64 & 2.12 \\
\hline 484 & Postal and Courier Services & 20 & 0.79 & 0.354 & 0.48 & 1.30 & 7 & 0.53 & 0.122 & 0.24 & 1.18 \\
\hline 4841 & Postal Service & 18 & 0.74 & 0.253 & 0.44 & 1.24 & 7 & 0.53 & 0.122 & 0.24 & 1.18 \\
\hline 49 & Other Utility & 34 & 0.95 & 0.782 & 0.66 & 1.37 & 18 & 1.11 & 0.692 & 0.66 & 1.86 \\
\hline 4911 & Electric Power Systems & 19 & 0.83 & 0.456 & 0.51 & 1.36 & 10 & 0.91 & 0.789 & 0.46 & 1.61 \\
\hline 51 & Petroleum Products, WH & 15 & 0.87 & 0.631 & 0.49 & 1.54 & 6 & 0.74 & 0.492 & 0.31 & 1.75 \\
\hline 521 & Food, Beverage, Drug and Tobacco, WH & 29 & 1.29 & 0.230 & 0.85 & 1.96 & 7 & 0.69 & 0.369 & 0.31 & 1.55 \\
\hline 55 & Motor Vehicle, Parts and Accessories, WH & 15 & 1.40 & 0.256 & 0.78 & 2.50 & 7 & 2.92 & 0.019 & 1.20 & 7.13 \\
\hline 56 & $\begin{array}{l}\text { Metal, Hardware, Plumbing, Heating, Building } \\
\text { Material, WH }\end{array}$ & 40 & 1.26 & 0.211 & 0.88 & 1.81 & 12 & 0.99 & 0.975 & 0.52 & 1.87 \\
\hline 563 & Lumber and Building Materials, WH & 25 & 1.24 & 0.334 & 0.80 & 1.92 & 11 & 1.44 & 0.297 & 0.73 & 2.86 \\
\hline 5631 & Lumber, Plywood and Millwork, WH & 15 & 1.37 & 0.278 & 0.78 & 2.42 & 5 & 1.23 & 0.679 & 0.46 & 3.28 \\
\hline 57 & Machinery, Equipment and Supplies, WH & 45 & 1.23 & 0.223 & 0.88 & 1.72 & 19 & 1.24 & $0.406 \mathrm{C}$ & 0.75 & 2.06 \\
\hline 579 & Other Machinery, Equipment and Supplies, WH & 12 & 0.99 & 0.975 & 0.53 & 1.83 & 5 & 1.26 & 0.639 & 0.48 & 3.31 \\
\hline 59 & Other Products, WH & 25 & 0.87 & 0.519 & 0.57 & 1.33 & 6 & 0.68 & 0.3800 & 0.29 & 1.61 \\
\hline 60 & Food, Beverage and Drugs, Retail & 55 & 0.85 & 0.291 & 0.63 & 1.15 & 23 & 1.01 & 0.9660 & 0.64 & 1.59 \\
\hline
\end{tabular}


Table 5. Cont

\begin{tabular}{|c|c|c|c|c|c|c|c|c|c|c|c|}
\hline \multirow{3}{*}{$\begin{array}{l}\text { Code } \\
601\end{array}$} & \multirow{3}{*}{$\begin{array}{l}\text { Industry Title } \\
\text { Food Stores }\end{array}$} & \multicolumn{5}{|c|}{ Ever } & \multicolumn{5}{|c|}{ Usual } \\
\hline & & \multicolumn{2}{|c|}{ Case OR } & \multirow{2}{*}{$\begin{array}{r}P \text { value } \\
0.597\end{array}$} & \multicolumn{2}{|c|}{$95 \%$ CI } & \multicolumn{2}{|c|}{ Case OR } & \multirow{2}{*}{$\frac{P \text { value }}{0.5910}$} & \multicolumn{2}{|c|}{$95 \% \mathrm{CI}$} \\
\hline & & 48 & 0.92 & & 0.68 & 1.25 & 19 & 1.15 & & 0.69 & 1.91 \\
\hline 6011 & Groceries Food Stores & 29 & 0.79 & 0.260 & 0.52 & 1.19 & 8 & 0.68 & 0.319 & 0.32 & 1.45 \\
\hline 6012 & Specialty Food Stores & 23 & 1.29 & 0.288 & 0.81 & 2.06 & 10 & 2.58 & 0.023 & 1.14 & 5.84 \\
\hline 62 & $\begin{array}{l}\text { Household Furniture, Appliances, Furnishing, } \\
\text { Retail }\end{array}$ & 22 & 0.98 & .933 & 0.61 & 1.57 & 5 & 0.59 & 0.267 & 0.23 & 1.50 \\
\hline 63 & $\begin{array}{l}\text { Auto Vehicle, Parts, Accessories, Sale and } \\
\text { Service }\end{array}$ & 86 & 0.95 & 88 & 0.74 & 1.22 & 28 & 0.81 & 0.324 & 0.53 & 1.23 \\
\hline 631 & Automobile Dealers & 23 & 1.17 & 0.497 & 0.74 & 1.84 & 11 & 1.40 & 0. & 0.71 & 2.77 \\
\hline 6311 & New) Dealers & 23 & 1.25 & 0.341 & 0.79 & 1.98 & 11 & 1.52 & 0.227 & 0.77 & 3.00 \\
\hline 635 & e Repair Shops & 48 & 0.96 & 0.806 & 0.69 & 1.33 & 13 & 0.72 & 0.273 & 0.40 & 1.30 \\
\hline 6351 & Motor Vehicle Repair Garage & 42 & 0.97 & 0.860 & 0.69 & 1.36 & 8 & 0.50 & 4 & 0.24 & 1.04 \\
\hline 64 & General Retail Merchand & 43 & 0.83 & 0.266 & 0.60 & 1.15 & 16 & 0.90 & 0.704 & 0.52 & 1.55 \\
\hline 641 & General Merchandise Stores & 43 & 0.83 & 0.266 & 0.60 & 1.15 & 16 & 0.90 & 0.704 & 0.52 & 1.55 \\
\hline 6411 & Department Stores & 36 & 0.91 & 0.612 & 0.63 & 1.31 & 14 & 1.02 & 8 & 0.56 & 1.85 \\
\hline 65 & Other Retail Stores & 36 & 0.93 & 0.703 & 0.64 & 1.35 & 9 & 0.64 & 0.202 & 0.32 & 1.27 \\
\hline 70 & Deposit Accepting I & 19 & 0.72 & 0.183 & 0.44 & 1.17 & 11 & 0.98 & 0.953 & 0.50 & 1.91 \\
\hline 702 & $\begin{array}{l}\text { Chartered Banks, Other Banking-Type } \\
\text { Intermediaries }\end{array}$ & 14 & 0.64 & 0.118 & 0.37 & 1.12 & 8 & 0.92 & 0.828 & 0.43 & 1.95 \\
\hline 7021 & Chartered Banks & 13 & 0.66 & 0.172 & 0.36 & 1.20 & 7 & 0.87 & 7 & 0.39 & 1.96 \\
\hline 73 & Insurance Underwriters & 27 & 1.63 & 0.032 & 1.04 & 2.55 & 9 & 1.12 & 0.759 & 0.54 & 2.31 \\
\hline 733 & Property and Casualty Ir & 14 & 1.46 & 0.219 & 0.80 & 2.67 & 7 & 1.51 & 0.342 & 0.65 & 3.53 \\
\hline 7339 & Other Property and Casualty Ins & 13 & 1.38 & 0.309 & 0.74 & 2.57 & 6 & 1.25 & 0.633 & 0.50 & 3.12 \\
\hline 76 & Insurance and Real Estate Agencies & 54 & 1.26 & 0.146 & 0.92 & 1.72 & 26 & 1.67 & 0.024 & 1.07 & 2.60 \\
\hline 77 & Business Services & 79 & 1.09 & 0.508 & 0.85 & 1.41 & 30 & 1.03 & 0.888 & 0.68 & 1.55 \\
\hline 773 & Accounting and Bookkeeping Services & 10 & 0.82 & 0.572 & 0.41 & 1.63 & 5 & 0.98 & 0.968 & 0.37 & 2.62 \\
\hline 775 & Architectural, Engineering and Other Services & 37 & 1.17 & 0.402 & 0.81 & 1.69 & 14 & 0.97 & 0.920 & 0.54 & 1.75 \\
\hline 7752 & Offices of Engineers & 20 & 1.20 & 0.473 & 0.73 & 1.98 & 6 & 0.75 & 0.519( & 0.31 & 1.80 \\
\hline
\end{tabular}


Table 5. Cont

\begin{tabular}{|c|c|c|c|c|c|c|c|c|c|c|}
\hline \multirow{3}{*}{$\begin{array}{l}\text { Code } \\
7759\end{array}$} & \multirow{3}{*}{$\begin{array}{l}\text { Industry Title } \\
\text { Other Scientific and Technical Services }\end{array}$} & \multicolumn{5}{|c|}{ Ever } & \multicolumn{4}{|c|}{ Usual } \\
\hline & & \multicolumn{2}{|c|}{ Case OR } & \multirow{2}{*}{$\frac{P \text { value }}{0.639}$} & \multicolumn{2}{|c|}{$95 \% \mathrm{CI}$} & \multicolumn{2}{|c|}{ Case OR } & \multirow{2}{*}{$\begin{array}{r}\boldsymbol{P} \text { value } \\
0.684\end{array}$} & $95 \%$ CI \\
\hline & & 16 & 1.14 & & 0.66 & 1.97 & 6 & 1.21 & & 3.03 \\
\hline 81 & Federal Government Services & 348 & 1.12 & 0.131 & 0.97 & 1.30 & 86 & 1.24 & 0.0990 .97 & 1.58 \\
\hline 811 & Defence Services & 316 & 1.11 & 0.168 & 0.96 & 1.29 & 66 & 1.41 & 0.0181 .06 & 1.88 \\
\hline 815 & General Administrative Service & 17 & 1.00 & 0.971 & 0.59 & 1.74 & 6 & 0.70 & 0.4170 .30 & 1.66 \\
\hline 817 & Economic Service Administration & 9 & 0.58 & 0.132 & 0.29 & 1.18 & 6 & 0.83 & 0.6750 .35 & 1.98 \\
\hline 82 & Provincial and Territorial Government Services & 45 & 1.11 & 0.538 & 0.80 & 1.55 & 20 & 1.24 & 0.4060 .75 & 2.06 \\
\hline 822 & Protective Services & 15 & 1.09 & 0.764 & 0.62 & 1.91 & 6 & 1.12 & 0.8030 .46 & 2.73 \\
\hline 826 & Human Resource Administrat & 10 & 2.34 & 0.024 & 1.11 & 4.89 & 7 & 4.72 & 0.0021 .79 & 12.46 \\
\hline 83 & Local Government Services & 33 & 0.84 & 0.345 & 0.59 & 1.20 & 18 & 0.96 & 0.8780 .57 & 1.62 \\
\hline 8323 & Police Services & 8 & 1.17 & 0.692 & 0.54 & 2.54 & 6 & 1.85 & 0.1930 .73 & 4.67 \\
\hline 8324 & Firefighti1 & 9 & 1.50 & 0.289 & 0.71 & 2.17 & 7 & 1.83 & 0.1680 .78 & 4.32 \\
\hline 85 & Educational Services & 88 & 1.05 & 0.704 & 0.82 & 1.35 & 48 & 1.01 & 0.9520 .73 & 1.40 \\
\hline 851 & Elementary and Secondary Education & 63 & 1.08 & 0.597 & 0.81 & 1.44 & 33 & 1.02 & 0.9190 .70 & 1.50 \\
\hline 853 & University Education & 21 & 1.17 & 0.524 & 0.72 & 1.90 & 11 & 1.07 & 0.8430 .55 & 2.09 \\
\hline 86 & Health and Social Servi & 55 & 0.98 & 0.896 & 0.72 & 1.33 & 28 & 0.98 & 0.9250 .65 & 1.49 \\
\hline 861 & Hospitals & 24 & 0.75 & 0.196 & 0.49 & 1.16 & 9 & 0.64 & 0.2240 .31 & 1.31 \\
\hline 8611 & General Hospitals & 18 & 0.68 & 0.124 & 0.42 & 1.11 & 6 & 0.47 & 0.0820 .20 & 1.10 \\
\hline 865 & $\begin{array}{l}\text { Offices: Physicians, Surgeons and Dentists, } \\
\text { Private }\end{array}$ & 15 & 1.31 & 0.368 & 0.73 & 2.36 & 13 & 1.27 & 0.4550 .68 & 2.38 \\
\hline 8651 & Offices: Physicians, General Practice & 7 & 1.48 & 0.371 & 0.63 & 3.49 & 6 & 1.57 & 0.3410 .62 & 3.98 \\
\hline 91 & Accommodation Services & 48 & 0.91 & 0.554 & 0.67 & 1.24 & 15 & 1.39 & 0.2590 .78 & 2.46 \\
\hline 911 & Hotels, Motels and Tourist Courts & 46 & 0.95 & 0.749 & 0.69 & 1.30 & 13 & 1.26 & 0.4640 .68 & 2.34 \\
\hline 9111 & Hotels and Motor Hotels & 35 & 0.88 & 0.488 & 0.61 & 1.26 & 9 & 1.09 & 0.8120 .54 & 2.22 \\
\hline 96 & Amusement and Recreational Servi & 27 & 0.97 & 0.888 & 0.64 & 1.48 & 6 & 0.97 & 0.9460 .40 & 2.36 \\
\hline 97 & Personal and Household Services & 19 & 0.85 & 0.522 & 0.52 & 1.40 & 9 & 0.93 & 0.8430 .46 & 1.90 \\
\hline 98 & Membership Organizations & 39 & 1.57 & 0.017 & 1.08 & 2.28 & 16 & 1.78 & 0.0580 .98 & 3.23 \\
\hline 981 & Religious Organizations & 15 & 1.17 & 0.595 & 0.66 & 2.09 & 8 & 1.20 & 0.6580 .54 & 2.69 \\
\hline 99 & Other Services & 27 & 0.65 & 0.040 & 0.43 & 0.98 & 8 & 0.69 & 0.3270 .33 & 1.45 \\
\hline
\end{tabular}




\subsection{Usual Industry (Table 5)}

In the usual industry category, a significant excess risk in the major industry group was noted at $\alpha=0.05$ for motor vehicle, parts and accessories, wholesale (SIC 55) and insurance and real estate agencies (SIC 76) and at $\alpha=0.10$ for beverage (SIC 11), federal government services (SIC 81) and membership organizations (SIC 98); ORs for printing, publishing and allied (SIC 28) industry was low at $\alpha=0.10$. Excess risks were significant at $\alpha=0.05$ for the following minor industry groups (three-digit codes): brewery products (SIC 113), defense services (SIC 811), and human resource administration (SIC 826 ) and at $\alpha=0.10$ for livestock farms (except animal specialties) (SIC 011), and dairy products (SIC 104). Odds ratio was significantly low at $\alpha=0.10$ for interior and finishing work (SIC 427). Several industry classes (four-digit codes) had significantly increased ORs at $\alpha=0.05$ for poultry and egg farms (SIC 0114), construction, mining machinery, materials handling (SIC 3192), exterior close-in: masonry work (SIC 4231), used goods moving and storage (SIC 4562), and specialty food stores (SIC 6012). ORs were significantly low at $\alpha=0.10$ for general freight trucking (SIC 4561), motor vehicle repair garages (general repairs) (SIC 6351) and general hospitals (SIC 8611).

In the validation study of the questionnaire, company job records of 81 individuals who reported having been employed in one of two large companies in $\mathrm{BC}$ were compared with information from their questionnaire; and the interclass correlation was 0.996 for starting year of employment and 0.971 for duration of employment.

\section{Discussion}

Significant associations between colon cancer and a number of occupations and industries were observed in our study. Several sedentary occupations in management, administration, bookkeeping and recording, financial management, insurance and real estate, and lodging and accommodation showed significantly increased risk of colon cancer either at $\alpha=0.05$ or at $\alpha=0.10$, which are consistent with findings from the previous studies [25-29].

In this study, we found some occupations and industries with significantly elevated risks of colon cancer in both ever and usual employment categories: workers working in occupations or industries with low physical activity, including insurance sales, human resource administration and membership organizations; rail transport equipment mechanics and repairers who were exposed to asbestos that has been reported to be associated with a higher risk of colon cancer; taxi drivers/chauffeurs who were exposed to engine exhaust and diesel engine emissions, a known carcinogen for colon cancer [21]; workers working in brewery products with exposure to chemicals such as ammonia refrigerant. Ammonia, potentially toxic to cells, shortens cell life span and alters DNA synthesis in various tissues including the ileum and colon [39] and is also thought to promote colon carcinogenesis in rats [40].

Elevated colon risks were also found in usual employment categories for occupations in wood products fabricating, assembling and repairing with exposure to wood dusts, a controversial agent without sufficient evidence of the association by the International Agency of Research on Cancer by far; industries of poultry and egg farms with exposure to gaseous agents such as ammonia from litter, carbon monoxide from poorly ventilated gas-fired heaters and hydrogen sulphide from liquid manure. 
Also, particles of organic or agricultural dust are aerosolized from poultry house litter; livestock farms (except animal specialties) and dairy products and relevant occupations with exposure to noxious gases such as hydrogen sulfide, and ammonia and methane in the ambient barn air; sawmill sawyers and workers working in motor vehicle parts and accessory wholesale and truck transportation on used goods, with exposure to engine exhaust and diesel engine emissions [21]; workers in construction, mining machinery material handling, brick and stone masons-tile setters as well as exterior close-in masonry work, with exposure to asbestos, which was widely used prior to 1978 in many building projects to increase the strength of concrete; ship engineering officers who were exposed to exhaust gases, engine emissions and asbestos; and workers in beverage industry with exposure to chemicals in gases and vapors typically involving chemical-handling activities related to cleaning operations, disinfection of process areas and use of preservatives in long-term food storage, in addition to thermal oils in the maintenance of heating and ammonia in cooling systems.

The strengths of this study are several folds. It is population-based with ascertainment of pathology confirmed cancer incident cases and includes lifetime occupational history with the ability to control for potential confounding factors and effect modifiers. Our study has revealed a number of occupational risk factors for colon cancer. Interpretation of those findings is limited by the lack of information on occupational exposures and the possibility that statistically significant results may have occurred by chance because of multiple comparisons; also, especially for many usual occupations and industries, numbers are few. In addition, other cancer cases used as controls may not be representative of the general population. Particularly, other cancers may also have some occupational risks that are common to those of colon cancer cases; in such situation, the corresponding estimated OR's noted in this study would underestimate the true risks. A missing value category was used in the analysis to avoid reducing the study sample size and that may yield biased results. However, since the proportion of missing data is small and it is unlikely that missing information on education and smoking is correlated with job classification, the biases if any should be relatively small. Some of the confounding factors such as measures of body-mass index and physical activities were not collected for this study. Nevertheless, our validation study demonstrates that self-reported employment history bear little recall errors in out study.

Some of the risks observed may not apply to current workers because of a potential decrease in industrial exposures. However, the results of our study are in line such as elevated colon cancer risk by exposure to asbestos and to engine exhaust and diesel engine emissions from the literature and further suggest that exposure to wood dusts and to ammonia may carry an increased risk of colon cancer. More specific studies using population controls are needed to investigate associations between occupation and exposures to chemical substances, taking into account changes in concentration levels over time.

\section{Acknowledgements}

The research was partially supported by grants from the Workers' Compensation of British Columbia and the National Health Research and Development Program, Health Canada. We gratefully acknowledge the invaluable contribution of the research assistants, Ms. Donna Kan and Ms. Barbara 
Jamieson. Finally, the authors would like to thank two anonymous reviewers and a guest editor who provided thoughtful and insightful comments that helped to improve this article.

\section{References}

1. Canadian Cancer Society/National Cancer Institute of Canada. Canadian Cancer Statistics 2008; Canadian Cancer Society/National Cancer Institute of Canada: Toronto, ON, Canada, 2008.

2. Keku, T.O.; Millikan, R.C.; Martin. C.; Rahkra-Burris, T.K.; Sandler, R.S. Family history of colon cancer: What does it mean and how is it useful? Am. J. Prev. Med. 2003, 24, 170-176.

3. Sharpe, C.R.; Siemiatycki, J.; Rachet, B. Effects of alcohol consumption on the risk of colorectal cancer among men by anatomical sub-site (Canada). Canc. Causes Contr. 2004, 13, 483-491.

4. Singh, P.N.; Fraser, G.E. Dietary risk factors for colon cancer in a low-risk population. Am. J. Epidemiol. 1998, 148, 761-774.

5. Giovannucci, E.; Rimm, E.B.; Stamfer, M.J.; Colditz, G.A.; Ascherio, A.; Willett, W.C. Intake of fat, meat, and fiber in relation to risk of colon cancer in men. Cancer Res. 1994, 54, 2390-2397.

6. Howard, R.A.; Freedman, D.M.; Park, Y.; Hollenbeck, A.; Schatzkin, A.; Leitzmann, M.F. Physical activity, sedentary behaviour, and the risk of colon and rectal cancer in the NIH-AARP diet and health study. Canc. Causes Contr. 2008, 19, 939-953.

7. Slattery, M.L.; Potter, J.D. Physical activity and colon cancer: Confounding or interaction? Med. Sci. Sports Exerc. 2002, 34, 913-919.

8. Thune, I.M.; Furberg, A.S. Physical activity and cancer risk: Dose response and cancer, all sites and site specific. Med. Sci. Sports Exerc. 2001, 33(Suppl. 6), S530-S550.

9. Macfarlane, G.J.; Lowenfels, A.B. Physical activity and colon cancer. Eur. J. Cancer Prev. 1994, 3, 393-398.

10. Johnsen, N.; Christensen, J.; Thomsen, B.L.; Olsen, A.; Loft, S.; Overvad, K.; Tjønneland, A. Physical activity and risk of colon cancer in a cohort of Danish middle-aged men and women. Eur. J. Epidemiol. 2006, 21, 877-884.

11. Frezza, E.E.; Wachtel, M.S.; Chiriva-Internati, M. Influence of obesity on the risk of developing colon cancer. Gut 2006, 55, 285-291.

12. Huang, X.-F.; Chen, J.-Z. Obesity, the PI3K/Akt signal pathway and colon cancer. Obes. Rev. 2009, 10, 610-615.

13. Larsson, S.C.; Wolk, A. Obesity and colon and rectal cancer risk: A meta-analysis of prospective studies. Am. J. Clin. Nutr. 2007, 86, 556-565.

14. Heineman, E.F.; Zahm, S.H.; McLaughlin, J.K.; Vaught, J.B. Increased risk of colorectal cancer among smokers: Results of a 26-year follow-up of US veterans and a review. Int. J. Cancer 1995, 59, 728-738.

15. Preventing Occupational Disease and Injury, 2nd ed.; Levy, B.S., Wagner, G.R., Rest, K.M., Weeks, J.L., Eds.; American Public Health Association: Washington, DC, USA, 2005.

16. Aliyu, O.A.; Cullen, M.R.; Barnett, M.J.; Balmes, J.R.; Cartmel, B.; Redlich, C.A.; Brodkin, C.A.; Barnhart, S.; Rosenstock, L.; Israel, L.; et al. Evidence for excess colorectal cancer incidence among asbestos-exposed men in the beta-carotene and retinol efficacy trial. Am. J. Epidemiol. 2005, 162, 868-878. 
17. Gamble, J.F. Asbestos and colon cancer: A weight-of-the-evidence review. Environ. Health Perspect. 1994, 102, 1038-1050.

18. De Roos, A.J.; Ray, R.M.; Gao, D.L.; Wernli, K.J.; Fitzgibbons, E.D. ; Ziding, F. ; Astrakianakis, G. ; Thomas, D.B.; Checkoway, H. Colorectal cancer incidence among female textile workers in Shanghai, China: A case-control analysis of occupational exposures. Canc. Causes Contr. 2005, 16, 1177-1188.

19. Ritz, B.; Zhao, Y.; Krishnadasan, A.; Kennedy, N.; Morgenstern, H. Estimated effects of hydrazine exposure on cancer incidence and mortality in aerospace workers. Epidemiology 2006, 17, 154-161.

20. Samanic, D.; Rusiecki, J.; Dosemeci, M.; Hou, L.; Hoppin, J.A.; Sandler, D.P.; Lubin, J.; Blair, A.; Alavanja, M.C. Cancer incidence among pesticide applicators exposed to dicamba in the Agricultural Health Study. Environ. Health Perspect. 2006, 114, 1521-1526.

21. Siemiatycki, J.; Gérin, M.; Stewart, P.; Nadon, L.; Dewar, R.; Richardson, L. Associations between several sites of cancer and ten types of exhaust and combustion products: Results from a case-referent study in Montreal. Scand. J. Work Environ. Health 1988, 14, 79-90.

22. Firth, H.M.; Herbison, G.P.; Cooke, K.R.; Fraser, J. Male cancer mortality by occupation: 1973-1986. New Zeal. Med. J. 1993, 106, 328-330.

23. Cerhan, J.R.; Cantor, K.P.; Williamson, K.; Lynch, C.F.; Torner, J.C.; Burmeister, L.F. Cancer mortality among Iowa farmers: Recent results, time trends, and lifestyle factors (United States). Canc. Causes Contr. 1998, 9, 311-319.

24. Goldberg, M.S.; Parent, M.E.; Siemiatycki, J.; Désy, M.; Nadon, L.; Richardson, L.; Lakhani, R.; Latreille, B.; Valois, M.F. A case-control study of the relationship between the risk of colon cancer in men and exposures to occupational agents. Am. J. Indus. Med. 2001, 39, 531-546.

25. Vetter, R.; Dosemeci, M.; Blair, A.; Wacholder, S.; Unsal, M.; Engin, K.; Fraumeni, J.F., Jr. Occupational physical activity and colon cancer risk in Turkey. Eur. J. Epidemiol. 1992, 8, 845-850.

26. Chow, W.H.; Dosemeci, M.; Zheng, W.; Vetter, R.; McLaughlin, J.K.; Gao, Y.T.; Blot, W.J. Physical activity and occupational risk of colon cancer in Shanghai, China. Int. J. Epidemiol. 1993, 22, 23-29.

27. Vena, J.E.; Graham, S.; Zielezny, M.; Swanson, M.K.; Barnes, R.E.; Nolan, J. Lifetime occupational exercise and colon cancer. Am. J. Epidemiol. 1985, 122, 357-365.

28. Steindorf, K.; Jedrychowski, W.; Schmidt, M.; Popiela, T.; Wahrendorf, J. Case-control study of lifetime occupational and recreational physical activity and risks of colon and rectal cancer. Eur. J. Cancer Prev. 2005, 14, 363-371.

29. Moradi, T.A.; Gridley, G.; Björk, J.; Dosemeci, M.; Ji, B.T.; Berkel, H.J.; Lemeshow, S. Occupational physical activity and risk for cancer of the colon and rectum in Sweden among men and women by anatomic sub-site. Eur. J. Cancer Prev. 2008, 17, 201-208.

30. Band, P.R.; Spinelli, J.J.; Threlfall, W.J.; Fang, R.; Le, N.D.; Gallagher, R.P. Identification of occupational cancer risks in British Columbia: I. Methodology, descriptive results, and analysis of cancer risks by cigarette smoking categories of 15,463 incident cancer cases. J. Occup. Environ. Med. 1999, 41, 224-232. 
31. World Health Organization (WHO). International Classification of Diseases, 9th Revision; WHO: Geneva, Switzerland, 1977.

32. World Health Organization (WHO). International Classification of Diseases for Oncology; WHO: Geneva, Switzerland, 1976.

33. Statistics Canada. Standard Occupational Classification 1980; Statistics Canada: Ottawa, ON, Canada, 1981.

34. Statistics Canada. Standard Industrial Classification 1980; Statistics Canada: Ottawa, ON, Canada, 1981.

35. Smith, A.H.; Pearce, N.E.; Callas, P.W. Cancer case-control studies with other cancers as controls. Int. J. Epidemiol. 1988, 17, 298-306.

36. Modern Epidemiology, 2nd edition; Rothman, K.J., Greenland, S., Eds.; Lippincott Williams \& Wilkins: Philadelphia, PA, USA, 1998.

37. Statistical Methods in Cancer Research, The Analysis of Case-Control Studies; Breslow, N.E., Day, N.E., Eds.; IARC Scientific Publications: Lyon, France, 1980; Vol. 1.

38. Storer, B.E.; Wacholder, S.; Breslow, N.E. Maximum likelihood fitting of general risk models to stratified data. Appl. Stat. 1983, 32, 177-181.

39. Visek, W.J. Diet and cell growth modulation by ammonia. Am. J. Clin. Nutr. 1978, 31, S216-S220.

40. Clinton, S.K.; Bostwick, D.G.; Olson, L.M.; Mangian, H.J.; Visek, W.J. Effects of ammonium acetate and sodium cholate on $N$-methyl-N9-nitro-Nnitrosoguanidine-induced colon carcinogenesis of rats. Cancer Res. 1988, 48, 3035-3039.

(C) 2011 by the authors; licensee MDPI, Basel, Switzerland. This article is an open access article distributed under the terms and conditions of the Creative Commons Attribution license (http://creativecommons.org/licenses/by/3.0/). 